\title{
Fundamental parameters and granulation properties of Alpha Centauri A and B obtained from inversions of their spectra ${ }^{\star}$
}

\author{
C. Frutiger ${ }^{1}$, S. K. Solanki ${ }^{2}$, and G. Mathys ${ }^{3}$ \\ 1 Institute of Astronomy, ETH-Zentrum, 8092 Zürich, Switzerland \\ 2 Max-Planck-Institut für Sonnensystemforschung ${ }^{\star \star}, 37191$ Katlenburg-Lindau, Germany \\ e-mail: solanki@mps.mpg.de \\ 3 European Southern Observatory, Casilla 19001, Santiago 19, Chile
}

Received 30 May 2005 / Accepted 25 August 2005

\section{ABSTRACT}

\begin{abstract}
Properties of stellar granulation are obtained by inverting spectra of the late-type stars $\alpha$ Centauri A and B. Our inversions are based on a multi-component model of the stellar photosphere and take into account the center-to-limb variation and rotational broadening. The different atmospheric components describe the areas harboring up-, down- and horizontal flows. The inversions are constrained by fitting not only the flux profiles, but also their line bisectors, and by using a simple mass conservation scheme. The inversions return the properties of convection at the stellar surface, including the stratification of the thermodynamic parameters, as well as fundamental parameters such as the gravitational acceleration, $v \sin i$ and the element abundances. For $\alpha$ Cen A (G2V) the derived stratifications of the temperature and convective velocity are very similar to the Sun, while for $\alpha$ Cen B (K1V) we find similar up- and downflow velocities, but lower horizontal speeds and a reduced overshoot. The latter is consistent with the smaller scale height of the atmosphere, while mass conservation arguments taken with the lower horizontal speed imply that the granules on $\alpha$ Cen B are smaller than on the Sun. Both these properties are in good agreement with the hydrodynamic simulation of Nordlund \& Dravins (1990, A\&A, 228, 155). The inversions also return the fundamental parameters $\left(T_{\text {eff }}, \log g\right.$, abundances, $v \sin i$, etc.) of the two stars. These values are on the whole in good agreement with literature values. Also, most of them do not strongly depend on the details of the inversion. However, importantly, the element abundances are 0.1 to 0.15 dex lower when a $2-$ or 3 -component inversion is carried out than with a 1-component inversion.
\end{abstract}

Key words. line: profiles - radiative transfer - stars: atmospheres - stars: late-type

\section{Introduction}

A remarkable feature of the solar envelope and other latetype stars is convection, which manifests itself in a patchwork of bright granular cells containing upflows and dark intergranular lanes harboring downflows. In the solar case, the photospheric absorption lines therefore show a typical blue-shift of about $0.5 \mathrm{~km} \mathrm{~s}^{-1}$ (after the the solar gravitational redshift of $0.636 \mathrm{~km} \mathrm{~s}^{-1}$ has been subtracted from the observed spectra; strong lines have more or less no line shift) and an asymmetric spectral profile. The latter is best described in terms of the line bisector showing a C-shape (cf. Dravins 1982, and references therein).

Convection at the solar surface has been studied extensively during the past decades (see, e.g., Spruit et al. 1990 for a review). Properties of the solar convection have been analyzed using numerical simulations (e.g. Nordlund \& Stein 1996; Stein \& Nordlund 1998; Asplund et al. 1999; Ploner et al. 2000), and numerous studies of data that sample only a

\footnotetext{
* Based on observations collected at the European Southern Observatories, La Silla, Chile.

$\star \star$ Known up to 30 June 2004 as the Max-Planck-Institut für Aeronomie.
}

small part of the solar disk (e.g. Hanslmeier et al. 1991; Nesis et al. 1993). Disk integrated spectra of the Sun (which treat the Sun as a star) have been investigated with the aim of studying granulation by Hamilton \& Lester (1998) and Livingston et al. (1999), while line profile inversions of such spectra have been carried out, e.g., by Allende Prieto et al. (1998) and Frutiger et al. (2000).

Several bright stars have been studied, too (see e.g. Gray 1998, 1999). One approach is based on the construction of parameterized theoretical models for which synthetic line profiles best fit the observations. Such models divide the stellar atmosphere into 2-4 components which describe the characteristic brightness and velocity of different surface fractions (cf. Gray \& Toner 1985; Dravins 1990).

Another approach is to construct a more consistent model of the photosphere and convection zone by solving the hydrodynamic equations (e.g. Nordlund \& Dravins 1990). Synthetic spectra calculated from these numerical simulations can be compared with the observations once the limited spatial resolution of the observations is taken into account. A limiting constraint of this type of models, however, is the large amount of computer time that they require. Also, not all types of stars have so far been amenable to this approach. 
Table 1. Stellar parameters from the literature.

\begin{tabular}{l|lll}
\hline \hline & $\alpha$ Cen A & $\alpha$ Cen B & Ref. \\
\hline $\mathrm{HR}$ & 5459 & 5460 & \\
$\mathrm{HD}$ & 128620 & 128621 & \\
$\mathrm{HIP}$ & 71683 & 71681 & \\
\hline$T_{\text {eff }}(\mathrm{K})$ & $5623\left(10^{3.75}\right)$ & $5248\left(10^{3.72}\right)$ & 1 \\
& $5800 \pm 20$ & $5320 \pm 50$ & 2 \\
& $5770 \pm 20$ & $5350 \pm 50$ & 8 \\
& $5710 \pm 25$ & - & 4 \\
$\log R / R_{\odot}$ & $0.11 \pm 0.01$ & $-0.07 \pm 0.00$ & 1 \\
$P_{\text {rot }}($ days $)$ & $23+5 /-2$ & $36.9 \pm 0.5$ & 6 \\
& 29 & - & 5 \\
$v \sin i\left(\mathrm{~km} \mathrm{~s}^{-1}\right)$ & $2.7 \pm 0.7$ & $1.1 \pm 0.8$ & 7 \\
{$[\mathrm{Fe} / \mathrm{H}]_{\odot}^{*}$} & $0.22 \pm 0.02$ & $0.26 \pm 0.04$ & 2 \\
& $0.12 \pm 0.02$ & - & 4 \\
$\log g$ & $4.22 \pm 0.05$ & $4.55 \pm 0.02$ & 1 \\
& $4.31 \pm 0.02$ & $4.58 \pm 0.02$ & 2 \\
& $4.27 \pm 0.02$ & - & 4 \\
& $4.4 \pm 0.1$ & $4.65 \pm 0.15$ & 3 \\
$v_{\text {mac }}\left(\mathrm{km} \mathrm{s}^{-1}\right)$ & $2.6 \pm 0.9$ & $1.0 \pm 0.8$ & 7 \\
\hline
\end{tabular}

References: 1 Allende Prieto \& Lambert (1999), Hipparcos catalogue; 2 Chmielewski et al. (1992); 3 Edvardsson (1988); 4 Furenlid \& Meylan (1990); 5 Hallam et al. (1991); 6 Jay et al. (1996); 7 Saar \& Osten (1997); 8 Soderblom (1986).

The stars belonging to the multiple system $\alpha$ Centauri have attracted considerable interest in the past two decades. Due to their brightness, $\alpha$ Cen A and $\alpha$ Cen B allow very high $S / N$ and high spectral resolution spectra to be recorded. The spectral type of $\alpha$ Cen A also makes it attractive for a comparison with the Sun. It can thus be used to test how well methods developed and tested on solar data (Frutiger et al. 2000) fare when applied to stellar spectra. For both stars the results obtained here can be compared with literature values.

In this work we evaluate stellar convection properties, for both $\alpha$ Cen A and $\alpha$ Cen B based on the simultaneous inversion of multiple photospheric absorption line profiles. The underlying model atmosphere is constructed from 2 or 3 components associated with stellar surface fractions of different characteristic brightness and velocity. The novelty of our approach is that we determine the stratification of temperature and velocity in each component of the atmosphere from an inversion of the spectra and thus overcome some of the limitations of previous models of this type. Line bisectors are also fit in addition to the line profiles themselves. In this way more weight is given to reproducing the relative wavelength shift and asymmetry of the line profiles, which are central diagnostics of granulation. In addition, a simple mass-conservation constraint takes into account the balance between mass flowing up and down at equal geometrical heights. This constraint allows the number of free parameters to be reduced, while at the same time imposing greater physical realism on the returned solutions. The same technique has been successfully applied to disk-integrated data of the Sun (Frutiger et al. 2000).

A selection of stellar parameters found in the literature is given in Table 1 together with their sources. In Table 2 we
Table 2. Best-fit parameters obtained by Dravins (1990) on the basis of 4-component models.

\begin{tabular}{|c|c|c|c|c|}
\hline \multirow{2}{*}{$\begin{array}{l}\text { Star } \\
\text { Lines }\end{array}$} & \multicolumn{2}{|c|}{$\alpha \operatorname{Cen~A}$} & \multicolumn{2}{|c|}{ Arcturus } \\
\hline & weak & strong & weak & strong \\
\hline Granular centers: & & & & \\
\hline Area $(\%)$ & 10 & 10 & 10 & 10 \\
\hline Brightness (\%) & 140 & 160 & 100 & 100 \\
\hline Velocity $\left(\mathrm{km} \mathrm{s}^{-1}\right)$ & -2.4 & -5.4 & -2.4 & -3.0 \\
\hline Granules: & & & & \\
\hline Area $(\%)$ & 20 & 20 & 20 & 20 \\
\hline Brightness (\%) & 120 & 130 & 100 & 100 \\
\hline Velocity $\left(\mathrm{km} \mathrm{s}^{-1}\right)$ & -1.2 & -2.7 & -1.5 & -2.1 \\
\hline Neutral areas: & & & & \\
\hline Area $(\%)$ & 55 & 55 & 50 & 50 \\
\hline Brightness (\%) & 100 & 100 & 100 & 100 \\
\hline Velocity $\left(\mathrm{km} \mathrm{s}^{-1}\right)$ & 0.0 & 0.0 & 0.0 & 0.0 \\
\hline Intergranular lanes: & & & & \\
\hline Area $(\%)$ & 15 & 15 & 20 & 20 \\
\hline Brightness (\%) & 47 & 20 & 100 & 100 \\
\hline Velocity $\left(\mathrm{km} \mathrm{s}^{-1}\right)$ & 3.3 & 7.2 & 2.7 & 3.6 \\
\hline
\end{tabular}

summarize the results of the 4-component model of Dravins (1990) in which the stellar surface is divided into granule centers, granules, neutral areas and intergranular lanes. The basic parameters of this model (for each component) are the dedicated surface area (filling factor), a depth independent velocity or line shift (which differ for weak and strong lines) and a brightness excess relative to the mean temperature of the neutral areas. Since he did not present a model for $\alpha$ Cen B we list the parameters of Arcturus (K1III) instead. These values may be compared with the detailed simulations carried out for $\alpha$ Cen B (K1V) (Nordlund \& Dravins 1990). We will compare these results with our findings in Sect. 4.

\section{Observations and atomic data}

The spectra used for our inversions are a subset of the data collected with the $1.4 \mathrm{~m}$ Coudé Auxiliary Telescope (CAT) of the European Southern Observatory and the Coudé Spectrograph (CES) in May 1988 (Rüedi et al. 1997). In order to assure that the whole data set for each star refers to the same rotational phase the individual $40 \AA$ wide spectral ranges with resolution $\approx 10^{5}$ and $S / N \approx 250$ were recorded on a single night for each star. The spectral ranges were primarily selected to contain a sufficient number of lines of interest for the magnetic field analysis by Rüedi et al. (1997). In the case of $\alpha$ Cen A and B, however, the maximum number of spectral ranges possible in a single night were recorded, in order to facilitate a detailed analysis of the stellar atmosphere. The wavelength was calibrated using as reference the lines of the star itself estimated to have roughly similar solar convective blueshifts (e.g. Dravins et al. 1981). These are in general lines of intermediate strength (cf. Allende Prieto et al. 2002). The laboratory wavelength values were assigned to the cores of these lines. A polynomial fit through multiple such lines was then used to assign a wavelength to each spectral point. 
Table 3. Atomic data of the inverted spectral lines.

\begin{tabular}{llccc}
\hline \hline$(1)$ & $(2)$ & $(3)$ & $(4)$ & $(5)$ \\
Ion & $\lambda_{\text {lab }}^{1}[\AA]$ & $\chi_{\mathrm{e}}[\mathrm{eV}]$ & $E^{2}$ & $\log g f$ \\
\hline $\mathrm{Fe}$ I & 5506.7791 & 0.9902 & 2.332 & $-2.797^{3}$ \\
$\mathrm{Fe}$ I & 5569.6181 & 3.4172 & 2.286 & $-0.540^{3}$ \\
$\mathrm{Fe}$ I & 5701.5446 & 2.5593 & 1.871 & $-2.216^{3}$ \\
$\mathrm{Fe}$ I & 6151.6181 & 2.1761 & 2.057 & $-3.299^{3}$ \\
$\mathrm{Fe}$ I & 6173.3356 & 2.2229 & 2.051 & $-2.880^{3}$ \\
$\mathrm{Fe}$ I & 6219.2810 & 2.1980 & 2.071 & $-2.433^{3}$ \\
$\mathrm{Fe}$ I & 6252.5554 & 2.4042 & 2.030 & $-1.687^{3}$ \\
$\mathrm{Fe}$ I & 6430.8464 & 2.1761 & 2.143 & $-2.006^{3}$ \\
$\mathrm{Fe} \mathrm{I}$ & 6750.1525 & 2.4244 & 2.161 & $-2.621^{3}$ \\
\hline $\mathrm{Fe}$ II & 5414.073 & 3.2215 & 2.0 & $-3.480^{4}$ \\
$\mathrm{Fe}$ II & 6149.258 & 3.8895 & 2.0 & $-2.724^{4}$ \\
$\mathrm{Fe}$ II & 6247.557 & 3.8919 & 2.0 & $-2.329^{4}$ \\
$\mathrm{Fe}$ II & 6432.680 & 2.8912 & 2.0 & $-3.510^{4}$ \\
\hline $\mathrm{Ca}$ I & 6162.1730 & 1.8991 & 2.343 & $-0.09^{5}$ \\
\hline
\end{tabular}

1 Nave et al. (1994), Kurucz \& Bell (1995).

2 Anstee \& O'Mara (1995), Barklem \& O'Mara (1997).

3 Fuhr et al. (1988), Kurucz \& Bell (1995).

4 Schnabel et al. (1999).

5 Miller et al. (1974), Kurucz \& Bell (1995).

For the analysis presented here we have selected several almost unblended lines of $\mathrm{Fe} \mathrm{I}$ and $\mathrm{Fe}$ II and the wings of the $\mathrm{Ca}$ I $6162 \AA$ line. The different iron lines, in particular those of Fe II, were selected to cover a wide range of excitation potentials and oscillator strengths with the goal to better sample the temperature and velocity at different levels in the atmosphere. The wings of the Ca I $6162 \AA$ line have been chosen for their sensitivity to the gravitational acceleration (cf. Edvardsson 1988; Cayrel et al. 1996). The LTE synthesis code, that lies at the heart of the inversion code, produces a very good approximation to the observed wings of this line, but not the core, which is affected by NLTE. Table 3 lists the adopted values of the important atomic parameters of all spectral lines employed for the inversions: Col. (2) gives the laboratory wavelengths. For Fe I lines they are taken from Nave et al. (1994), for Fe II they are treated as free parameters following Bellot Rubio et al. (1999) to overcome problems related to their generally less accurate values. Column (3) lists the excitation energy of the lower level of the transition, taken from Sugar \& Corliss (1985). The collisional damping parameters used for the calculation of the synthetic line profiles are derived from the cross-sections published by Anstee \& O'Mara (1995), Barklem \& O'Mara (1997) and Barklem et al. (1998) except for the values for Fe II which are estimated values. Column (4) shows the corresponding enhancement factors $E$ (at $T=5000 \mathrm{~K}$ ) over the values of the damping constant calculated using the simple Unsöld formula for Van der Waals broadening (Unsöld 1955). Column (5) lists the oscillator strengths, $\log g f$, taken from the literature, along with the sources.

\section{Inversion code and underlying model atmospheres}

The inversion code used for this analysis is a successor of the code used for the magnetic field analysis of active cool stars by Rüedi et al. (1997). It includes a new way of modeling depth-dependent physical quantities and has recently been extended by a Hermitian method for the solution of the radiative transfer equation (similar to Bellot Rubio et al. 1998) and response functions for an efficient computation of derivatives of spectral line profiles with respect to the free parameters (cf. Landi Degl'Innocenti 1976; Ruiz Cobo \& Del Toro Iniesta 1992).

The same inversion technique presented here has been applied to quiet-sun data (observed at solar disk center) and tested for full-disk solar spectra. I.e. data of the Sun as a star (Frutiger et al. 2000). In the sequel we summarize the basic concepts of the stellar model atmosphere underlying the inversions.

In our models the stellar surface is divided into 2 , respectively 3 different areas harboring an upflow, a downflow and a horizontal flow (present in 3-component models only). Each component is represented by a simple plane-parallel atmosphere for which the basic physical quantity, the temperature stratification, is specified by a set of free depth-dependent parameters. To be more precise, the temperature in the upflow, $T_{\text {up }}$, and downflow, $T_{\mathrm{dn}}$ are given on a coarse depth grid of 6 points $\left(\log \tau_{5000}=-4.5,-3,-2,-1,-0.2,0.6\right.$, where $\tau_{5000}$ is the optical depth at $5000 \AA$ ). From this coarse grid the stratifications are interpolated using splines under tension onto a fine depth grid $\left(\Delta \tau_{5000}=0.05\right)$. They are also extrapolated to both smaller and larger $\log \tau_{5000}$ values, up to a largest $\log \tau$ value of 1.5 and a smallest value of -6 . The latter grid is used for solving the radiative transfer equations. The third component, i.e. the component harboring a horizontal flow is treated differently. In order to keep the number of free parameters reasonably small, its temperature is given by

$T_{\text {hor }}=(1-\beta) \cdot T_{\text {up }}+\beta \cdot T_{\mathrm{dn}}$,

with $0<\beta<1$ the only additional free parameter. Physical quantities like the gas pressure, electron pressure, density and continuum opacity are derived assuming LTE and hydrostatic equilibrium.

The line-of-sight velocity of the downflow component is parameterized very similarly to the temperature, i.e. using 6 free parameters for the speeds at different optical depths. The (granular) upflow velocity is derived from the already defined quantities as follows:

$\alpha_{\text {up }} \cdot \rho_{\text {up }}(z) \cdot v_{\text {up }}(z)=\alpha_{\mathrm{dn}} \cdot \rho_{\mathrm{dn}}(z) \cdot v_{\mathrm{dn}}(z)$

with $\rho_{\text {up }}\left(\rho_{\mathrm{dn}}\right)$ being the density in the up- and downflow component, respectively, and $v_{\text {up }}\left(v_{\mathrm{dn}}\right)$ the corresponding velocity perpendicular to the stellar surface. This constraint assures that at each geometrical height in the atmosphere the mass flowing up equals the mass flowing down. The treatment of the horizontal velocity is described below.

Two important free parameters are the filling factors $\alpha_{\text {hor }}$ and $\alpha_{\mathrm{dn}}$. They determine the fractional areas harboring either a horizontal flow $\left(\alpha_{\text {hor }}\right)$, a downflow $\left(\alpha_{\mathrm{dn}}\right)$ or an upflow $\left(\alpha_{\text {up }}=1-\alpha_{\text {hor }}-\alpha_{\text {dn }}\right)$. Other free parameters are the gravitational acceleration, $\log g$, the depth-independent micro-turbulence in the up- and downflow component, $v_{\text {mic,up }}$ and $v_{\text {mic,dn }}\left(v_{\text {mic,hor }}=\right.$ $\left.\left(v_{\text {mic,up }}+v_{\text {mic,dn }}\right) / 2\right)$, the elemental abundances, $A_{\mathrm{Fe}}$ and $A_{\mathrm{Ca}}$, the 


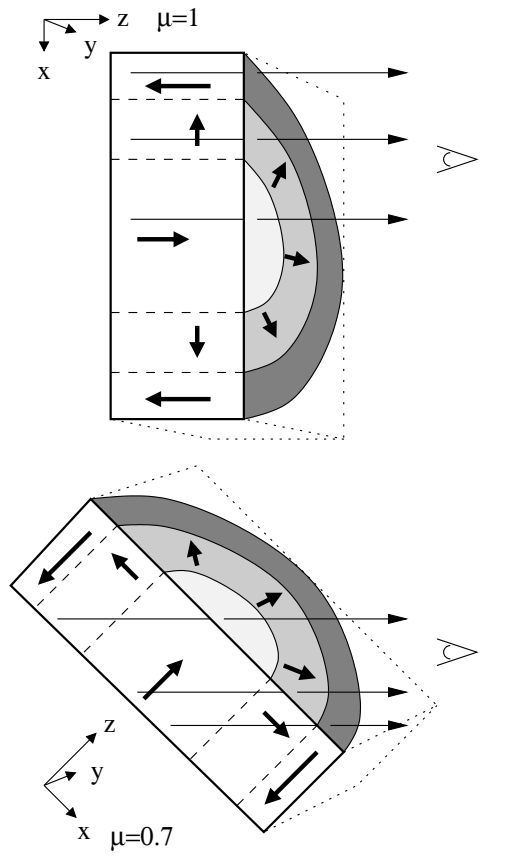

Fig. 1. Cut through a schematic 3-component model of a cylindrical granular cell observed at $\mu=1$ and $\mu=0.7$. Thick arrows refer to the components with an upflow (granule), downflow (intergranular lanes) and a horizontal flow. The three components are distinguished on the stellar surface by the shading with the bright upflows, denoted by the lightest gray and the dark downflows being shaded the heaviest. The arrows in the horizontal component indicate the radial outflow of material. At disk center $(\mu=1)$ the different surface fractions harbor either a purely longitudinal (vertical) flow or transverse (horizontal) flows. Towards the limb the treatment of the 3-dimensional structure of the granular flow field gains in complexity. For example the predominately horizontal flows (intermediate gray surface) have a varying but in general non-vanishing line-of-sight component which must to be taken into account. For more details see Sect. 3 .

radial and tangential macro-turbulence, $v_{\text {mac,rad }}$ and $v_{\text {mac,tan }}$ (see below), and an absolute velocity shift, $v_{\text {abs, }}$, which takes into account that the zero point of the wavelength scale may not be perfectly accurate. A last free parameter is the stellar rotation parameter $v \sin i$.

The disk-integrated synthetic intensity line profiles that are fit to the observations are obtained as a summation of line profiles from the different components each calculated at 9 "optimal" $\mu$ values (S. Saar, private communication; $\mu=\cos \theta$ with $\theta$ the angle between the line of sight and the surface normal), appropriately broadened (see below) and weighted for their surface coverage. The $\mu$ values are optimal in the sense that the flux profile obtained from only 9 intensity profiles is a good approximation to a flux profile resulting from a large number of intensity profiles at different $\mu$.

The idea of our 2(3)-component models is illustrated in Fig. 1 for a cylindrical granule. At disk center the vertical velocity corresponds to the line-of-sight velocity (responsible for the line shifts), while the macro-turbulence $\left(v_{\text {mac,rad }}\right)$ takes into account the spread in the line-of-sight component of a realistic velocity field (and is responsible for the line broadening). Towards the limb, however, this simple model becomes increasingly inadequate for describing the horizontal flows. As indicated by the thick arrows the horizontal flow field (parallel to the surface) points radially away from the granule center toward the intergranular lanes ${ }^{1}$. The net effect is thus to broaden the line profiles, but not to shift them, which is very similar to a macro-turbulent broadening. By using Gray's radial-tangential function (cf. Gray 1975, 1992, Chap. 18) we approximate the Doppler shift distribution $\Theta$ by

$\Theta(\Delta \lambda)=A_{\mathrm{R}} \Theta_{\mathrm{R}}(\Delta \lambda)+A_{\mathrm{T}} \Theta_{\mathrm{T}}(\Delta \lambda)$

where $A_{\mathrm{R}}$ and $A_{\mathrm{T}}$ are the stellar surface fractions presumed to have a radial, respectively tangential motion and $\Theta_{\mathrm{R}}=\Theta\left(v_{\text {mac,rad }}\right), \Theta_{\mathrm{T}}=\Theta\left(v_{\mathrm{mac}, \text { tan }}\right)$ the corresponding Gaussian Doppler shift distributions. For the 1- and 2-component model we have set $A_{\mathrm{R}}=A_{\mathrm{T}}=0.5$. This is a reasonable, although not unique choice. For the 3-component models we have used $A_{\mathrm{R}}=1-\alpha_{\text {hor }}$ and $A_{\mathrm{T}}=\alpha_{\text {hor }}$ (i.e. the same area fraction as harbours horizontal flows also contributes to the tangential turbulent velocity broadening).

The synthetic profiles are finally convolved with a Gaussian $\left(\sim \exp \left[\left(\xi / \xi_{\text {inst }}\right)^{2}\right], \xi=c \Delta \lambda / \lambda\right)$ of width $\xi_{\text {inst }}=1.3 \mathrm{~km} \mathrm{~s}^{-1}$ in order to take into account the instrumental broadening. Since the shape of the profile to model the instrumental as well as the macroturbulence broadening are not certain, the macroturbulence values obtained in our analysis must be taken with care.

\section{Results}

The $\alpha$ Cen A and B spectra have been inverted a number of times using 1-, 2- and 3-component models and a variety of constraints. The 1-component model has been used to get an average stratification of the stellar atmospheres for comparison purpose, while the multi-component models are employed to deduce the unresolved granulation properties. In order to obtain good fits in the 1-component model the wavelength shift of each spectral line is a free parameter, while in the 2- and 3-component models all lines are shifted by the same velocity stratification. For 13 fitted lines the 1-component model actually has more free parameters than the 2-component and 3-component models.

Best-fit intensity profiles resulting from a 3-component model and line bisectors together with the observed data for $\alpha$ Cen A and its companion $\alpha$ Cen B are depicted in Figs. 2 and 3, respectively. The differences between the observed and synthetic intensity profiles are generally below $2 \%$ of the observed intensities. The fits to the CaI wings (formally treated as two separate lines with identical properties) are particularly good and confirm the usefulness and accuracy of the new damping cross sections published by Anstee \& O'Mara (1995).

The fits to the observed bisectors are satisfactory except for the Fe I 6252.5 line, for which the discrepancy may be due to a small error in the laboratory reference wavelength. Our treatment of the Ca I wings did not allow the bisectors of this line to be fit. Since the bisectors of the remaining Fe I lines in row $\mathrm{d}$ are fit as accurately a those of the other lines they

\footnotetext{
1 The actual shape of the granules does not play a role, only the fractional areas enter the calculations.
} 

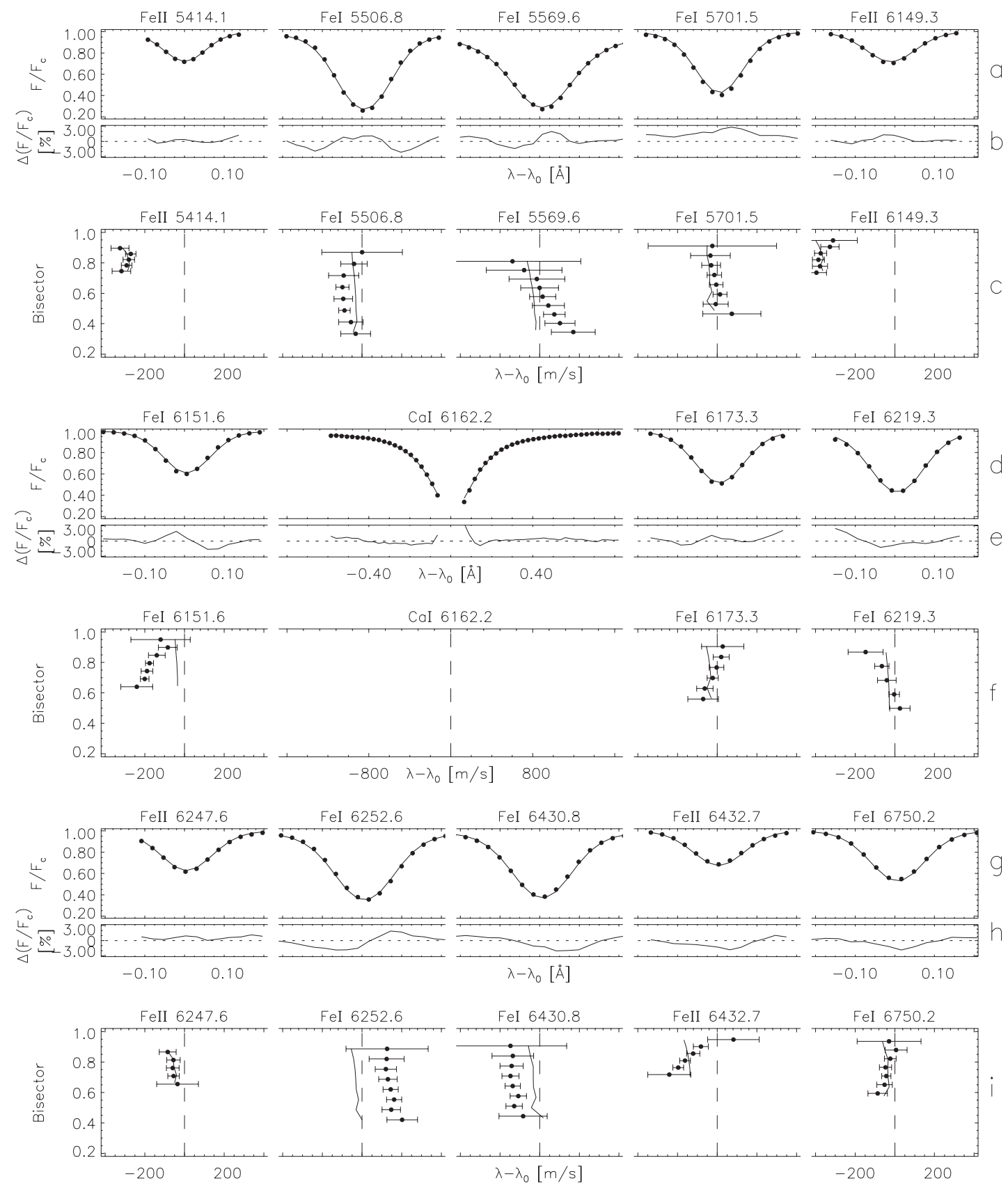

Fig. 2. Observed and best-fit relative flux $F / F_{\mathrm{c}}$ profile and line bisectors of $\alpha$ Cen A (here $F_{\mathrm{c}}$ is the continuum flux). The best-fit profiles result from a 3-component model. In rows a, $\mathrm{d}$ and $\mathrm{g}$ thin curves refer to the synthetic spectra, dots to the observed spectra of $\alpha$ Cen A. The rows $\mathrm{b}$, e and $\mathrm{h}$ show the differences between the synthetic and observed profiles on a scale stretched by a factor 100 for clarity. The dotted horizontal lines in these frames indicate a vanishing difference, i.e. a perfect fit. Rows c, f and i show the observed (dots) and synthetic (lines) line bisectors. The error bars give the expected uncertainties derived from those of the observed $F / F_{\mathrm{c}}$ profiles. The dashed vertical lines indicate the reference wavelength. See Sect. 4.

are not shown either. The bisectors shown in Figs. 2 and 3 are normalized to the line-depth, i.e. the bottom of the absorption line profile corresponds to position zero on the vertical axis.

The bisectors, both observed and calculated, are relatively straight and do not, in most cases, show a clear "C" shape. Partly this is due to the fact that disk-integrated starlight is being analyzed. As can be seen by comparing Figs. 5 and 8 of Frutiger et al. (2000), in which line bisectors obtained from the spectral atlases of Neckel $(1994,1999)$ are plotted, the disk integrated solar line bisectors are far less curved than the disk centre bisectors. In addition, bisectors extracted from spectra recorded at lower spectral resolution are less curved than those obtained at higher spectral resolutions (Dravins 1987a). Finally, as pointed out by Dravins (1987b), even at high spectral resolution, the bisector can vary significantly from one line to another due to blends and noise. In spite of these restrictions the results returned by the inversions for $\alpha$ Cen A agree well with solar results (see below). 

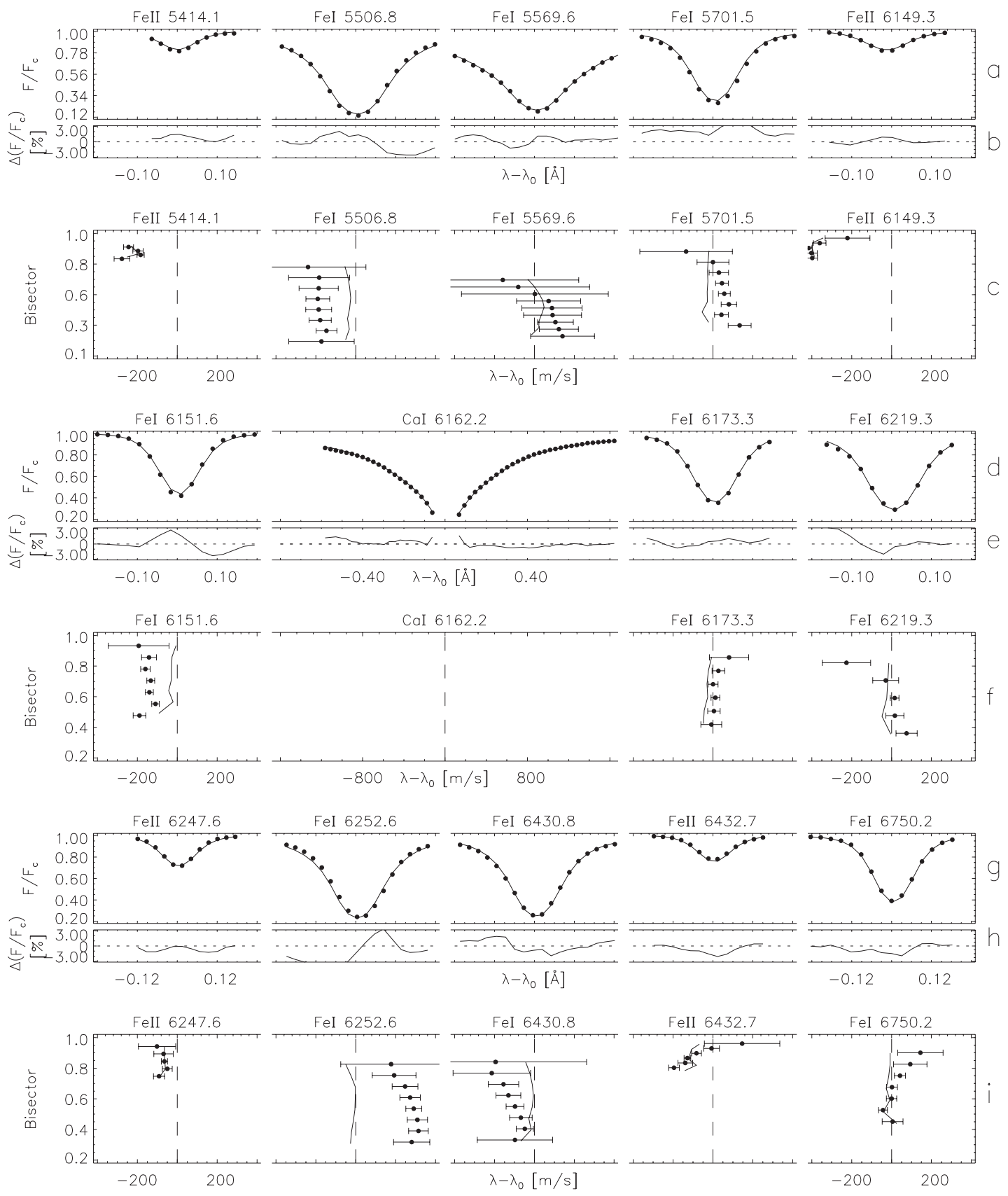

Fig. 3. Same as Fig. 2 but for $\alpha$ Cen B.

Temperature and velocity stratifications obtained from the inversions are shown in Figs. 4-7, while the best-fit values of the remaining (depth-independent) free parameters are listed in Tables 4 and 5. Column (1) of Table 4 lists the number of components used in the model atmosphere. The next two columns give the effective temperature obtained from the average atmosphere. The values in Col. (2) have been calculated from weighted fluxes by the simple formula (grey atmosphere, Edington-Barbier relation)

$T_{\text {eff,EB }}=\left[\sum \alpha_{k} T_{k}^{4}(\tau=2 / 3)\right]^{1 / 4}, \quad k=$ up, dn, hor

where $\tau$ is the optical depth, $\alpha_{k}$ are filling factors and $T_{k}$ the temperatures. The Rosseland opacity is used to approximate the $\tau$ of a grey atmosphere. The next column gives the effective temperature derived from flux spectra of each component computed using the ATLAS9 code (Kurucz 1970) with our best-fit temperature stratifications as input atmospheres $(T(\tau)$, $P_{e}(\tau)$ and $P_{g} a s(\tau)$ were fed to the atlas code). $T_{\text {eff, kur }}$ is thus obtained by first adding together filling factor weighted flux spectra (each covering the wavelength range $9 \mathrm{~nm}-160000 \mathrm{~nm}$ ) of all components and then integrating over all wavelength. This somewhat complex procedure was adopted due to the fact, that the temperature stratification of the deduced atmospheres differed considerable from radiative equilibrium of a grey atmosphere, so that the use of the Edington-Barbier relationship underlying $T_{\text {eff,EB }}$ is questionable. Column (4) lists the logarithm of the gravitational surface acceleration, Col. (5) the projected stellar rotation velocity. The next two columns give the 


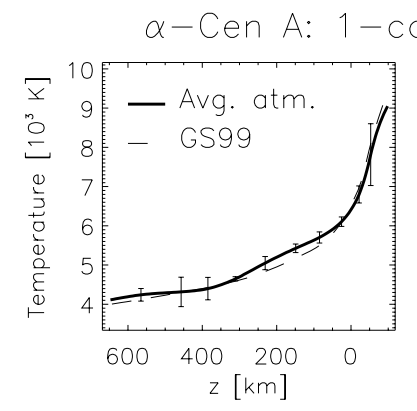

$\alpha$-Cen A: 2-component model
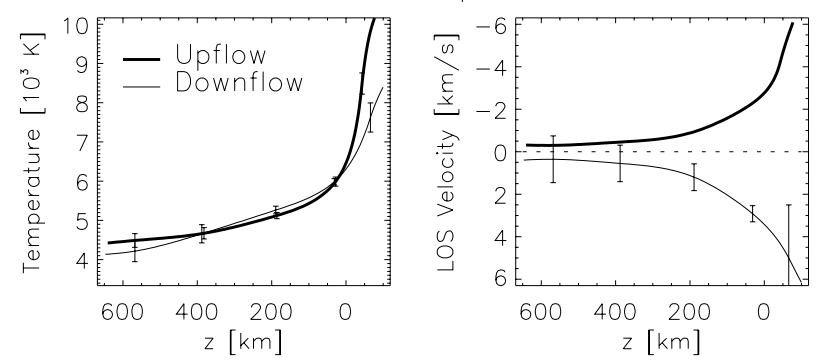

$\alpha$-Cen A: 3-component model
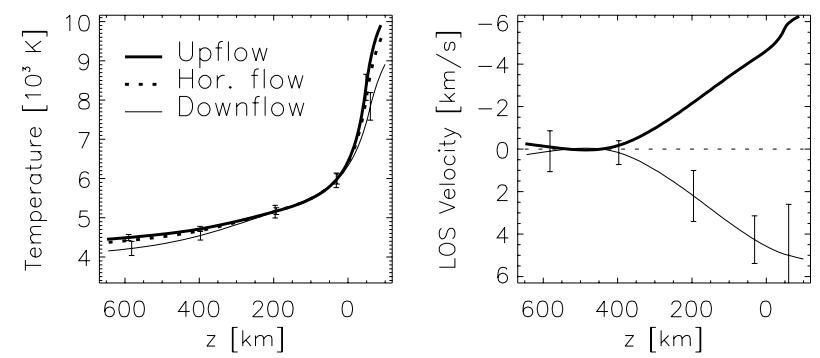

Sun: 3-component model
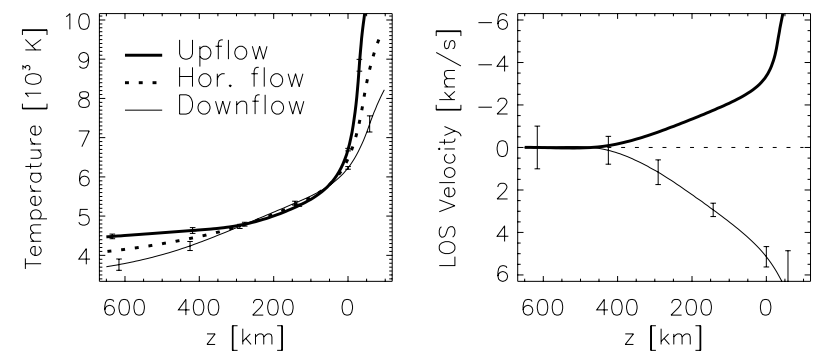

Fig. 4. Best-fit temperature and velocity stratifications (solid curves) for $\alpha$ Cen A. The first three rows shows the results obtained from inversion based on 1, 2 and 3 components, respectively. In the 1-component model no velocities are fit, but individual wavelengthshifts are allowed for each spectral line. The dashed curve represent the temperature stratification of the solar photospheric model of Grevesse \& Sauval (1999). The bottom row depicts the results obtained for the Sun by Frutiger et al. (2000). Error bars are shown at the depths at which free parameters are located. Note that since the upflow speed is obtained from the downflow through mass conservation, it is not independently determined, so that no error bars have been added.

elemental abundances on the usual logarithmic scale for the two elements for which absorption profiles are inverted $\left(A_{\mathrm{Fe}, \odot} \approx 7.5, A_{\mathrm{Ca}, \odot} \approx 6.36\right)$. Columns (8) and (9) give the fractional area harboring an upflow, $\alpha_{\text {up }}$, and downflow, $\alpha_{\mathrm{dn}}$, respectively. Finally, the last column lists the normalized $\chi^{2}$ of
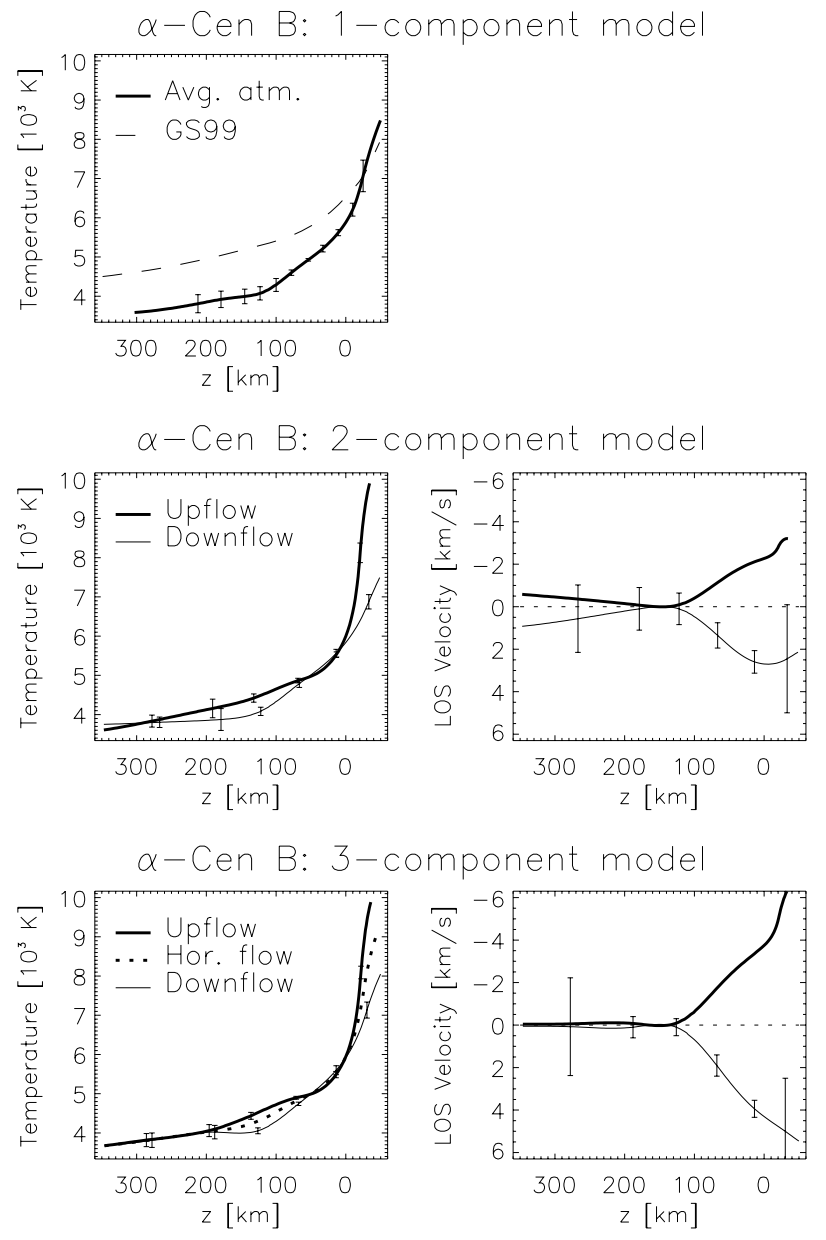

Fig. 5. Same as Fig. 4 but for best-fit temperature and velocity stratifications for $\alpha$ Cen B.

the inversions. A similarly small value means that the fits are equally good, irrespective of the number of data points (number of spectral lines) or number of free parameters (see Frutiger et al. 2000). Parameters that have been fixed at a given input value are indicated by italic fonts.

In Table 5 Cols. (2) and (3) list the depth-independent micro-turbulent velocity in the up- and downflow areas. The micro-turbulent velocity in the area harboring a horizontal flow is taken as the average of these two quantities. The last two columns give the macro-turbulent velocities used to describe the spread in the line-of-sight components of a realistic granular flow field and to take into account limb effects in the horizontal flows, as described above.

Next we discuss the results obtained for each star individually.

\section{1. $\alpha$ Cen A}

The similarity between the temperature and velocity structure of $\alpha$ Cen A and those of the Sun is striking (Fig. 4). At the continuum forming layers the inversions clearly reveal a hot area harboring an upflow and a cool area of similar size harboring a downflow. Higher up there is a weak inversion in the temperature contrast, exactly as for the Sun taking into account the 

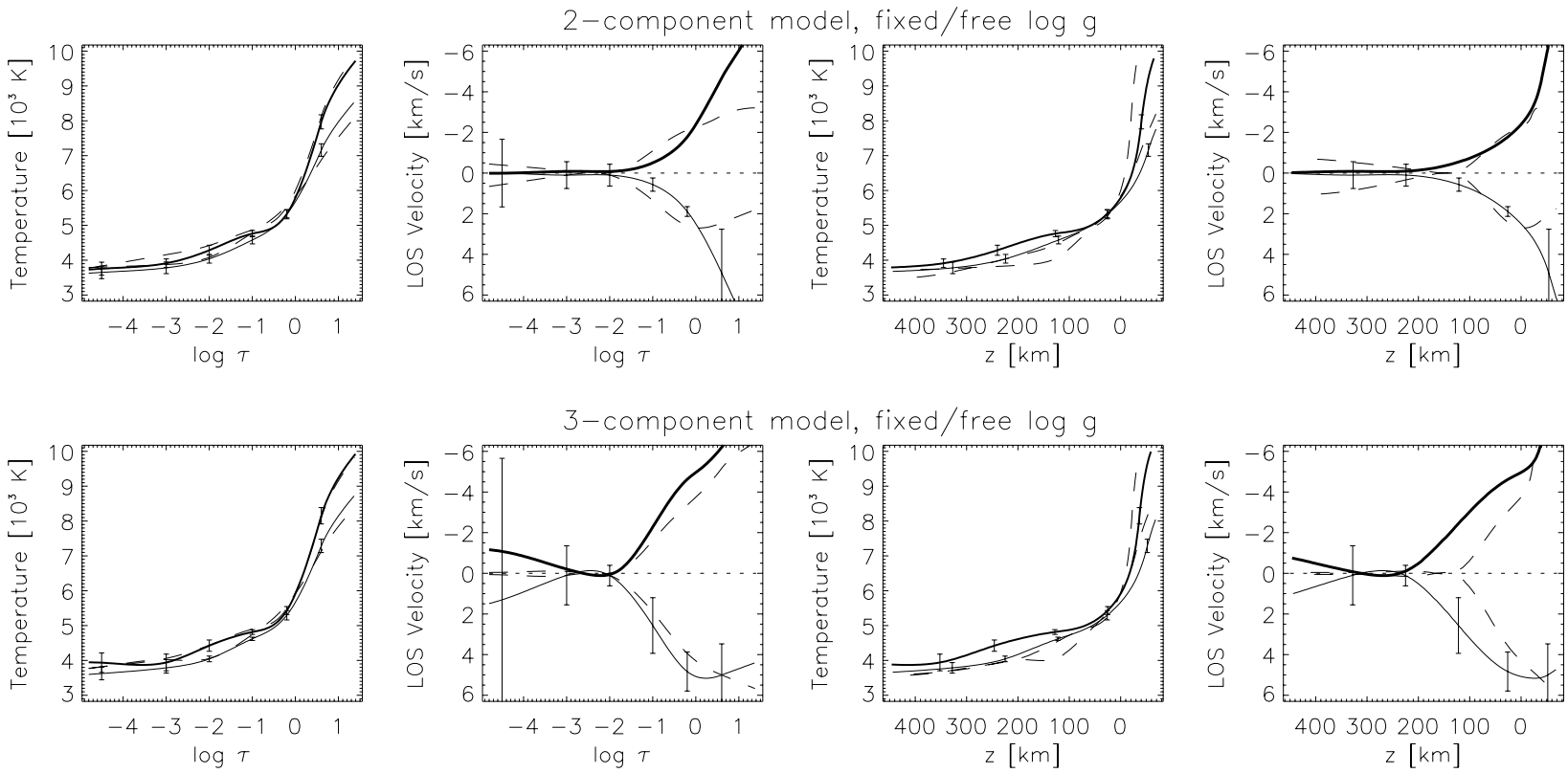

Fig. 6. Similar to Fig. 5, but for best-fit temperature and velocity stratifications obtained from inversions with fixed $\log g$ (solid, $\log g=4.48$, as given by Allende Prieto \& Lambert 1999) and free $\log g$ (dashed, as shown in Fig. 5). The stratifications are given on the logarithmic optical depth scale $\log \tau_{5000}$ (left two columns) and on the geometrical height scale (right two columns). Results obtained from the 2-component model are shown in the upper row, those from the 3-component model in the lower row. For clarity the temperature stratifications for the horizontal velocity component are not plotted. For the same reason the dashed curves are plotted without error bars. These may be found in Fig. 5 .
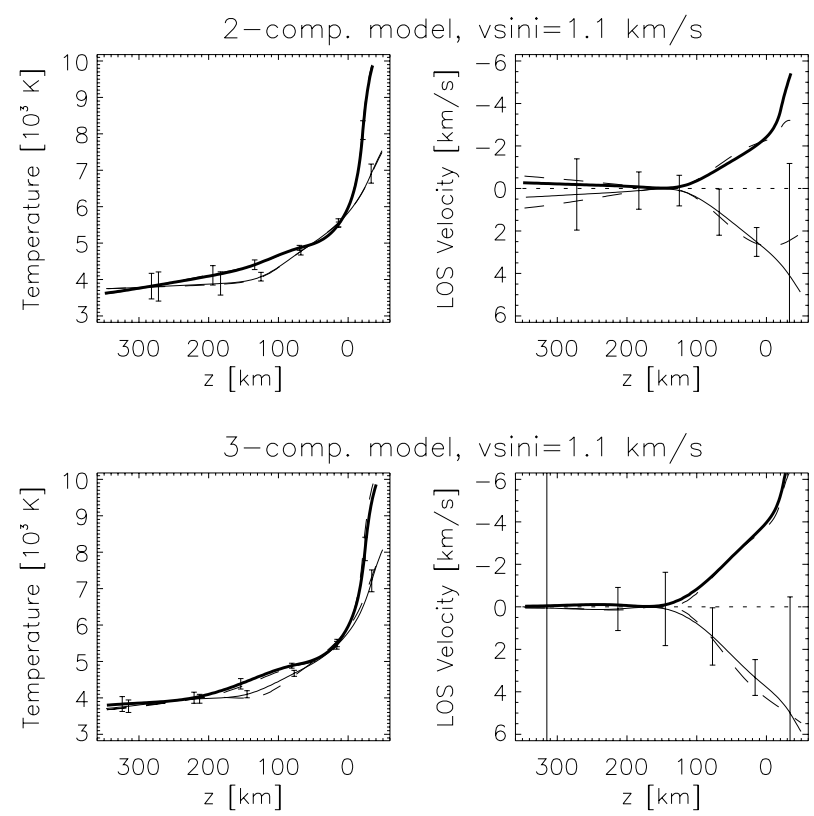

Fig. 7. Best-fit temperature and velocity stratifications for $\alpha$ Cen B returned by the inversion code for the case that the stellar rotation parameter $v \sin i$ is fixed to $1.1 \mathrm{~km} \mathrm{~s}^{-1}$ (solid) and free $v \sin i$ (dashed, as shown in Fig. 5). The upper row shows the results obtained using the 2-component model, the lower rows those for the 3-component model.

given error bars, one can state that at the higher layers the temperatures of the up- and downflow components are the same). The effective temperature obtained from the multi-component inversions is smaller than the solar value and close to the value found by Furenlid \& Meylan (1990). The logarithmic value given by Allende Prieto \& Lambert (1999) derived from data of the the Hipparcos mission also indicates a cooler surface for $\alpha$

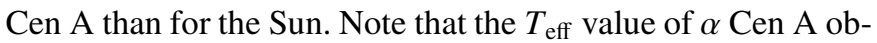
tained from the 2- and 3-component models is approximately $200 \mathrm{~K}$ smaller than from 1-component inversions. This corresponds roughly to a $1 \sigma$ difference.

The $\log g$ and $v \sin i$ values returned by the inversion are in good agreement with the values found in the literature (compare Tables 1 and 4). The excess in metallicity compared with the solar value, $A_{\mathrm{Fe}, \odot}=7.5 \pm 0.05$ (Grevesse \& Sauval 1999), is considerably larger for the 1-component average atmosphere than for the models taking into account the granulation. The lower metal abundances obtained by multicomponent models are compatible with the results obtained by using 3-D hydrodynamic simulations (e.g. Asplund et al. 2005, and references therein). The spread in the results reflects the differences of previous findings (see Table 1) and clearly shows the dependence of the derived abundances from the atmospheric models. We believe that the lower abundances obtained employing the multi-component models are the more reliable ones.

The granular velocity structure returned by the inversions depends on the number of components used in the underlying model atmosphere (Fig. 4). When using only an up- and downflow component the spectral contributions from areas harboring a flow directed parallel to the surface is accounted for by a larger macro-turbulent broadening while the up- and downflow velocities are reduced. This is what we expect when we assume that near the disk center, which contributes most to the total flux, the unshifted absorption in areas harboring a mainly horizontal flow is compensated for by more broadened 
Table 4. Best-fit fundamental parameters.

\begin{tabular}{|c|c|c|c|c|c|c|c|c|c|}
\hline (1) & $(2)$ & (3) & (4) & (5) & (6) & (7) & (8) & (9) & (10) \\
\hline $\begin{array}{c}\text { Star } \\
\text { \#comp }\end{array}$ & $\begin{array}{c}T_{\text {eff,EB }} \\
(\mathrm{K})\end{array}$ & $\begin{array}{c}T_{\text {eff, Kur }} \\
(\mathrm{K})\end{array}$ & $\log g$ & $\begin{array}{c}v \sin i \\
\left(\mathrm{~km} \mathrm{~s}^{-1}\right)\end{array}$ & $A_{\mathrm{Fe}}$ & $A_{\mathrm{Ca}}$ & $\alpha_{\text {up }}$ & $\alpha_{\mathrm{dn}}$ & $\chi^{2}$ \\
\hline \multicolumn{10}{|l|}{$\alpha$ Cen A } \\
\hline 1 & $5870 \pm 217$ & 5810 & $4.38 \pm 0.06$ & $1.5 \pm 0.8$ & $7.76 \pm 0.02$ & $6.56 \pm 0.02$ & - & - & 5.0 \\
\hline 2 & $5680 \pm 160$ & 5712 & $4.29 \pm 0.07$ & $2.2 \pm 0.4$ & $7.60 \pm 0.04$ & $6.39 \pm 0.04$ & $0.56 \pm 0.03$ & $0.44 \pm 0.03$ & 5.4 \\
\hline 3 & $5664 \pm 130$ & 5705 & $4.28 \pm 0.03$ & $2.9 \pm 0.7$ & $7.58 \pm 0.02$ & $6.38 \pm 0.02$ & $0.19 \pm 0.06$ & $0.17 \pm 0.06$ & 5.8 \\
\hline \multicolumn{10}{|l|}{$\alpha$ Cen B } \\
\hline 1 & $5394 \pm 165$ & 5327 & $4.86 \pm 0.05$ & $1.53 \pm 2.3$ & $7.69 \pm 0.03$ & $6.57 \pm 0.04$ & - & - & 10.5 \\
\hline 2 & $5267 \pm 20$ & 5313 & $4.75 \pm 0.05$ & $0.1 \pm 1.9$ & $7.56 \pm 0.02$ & $6.42 \pm 0.03$ & $0.55 \pm 0.08$ & $0.45 \pm 0.08$ & 10.5 \\
\hline 3 & $5256 \pm 67$ & 5310 & $4.74 \pm 0.02$ & $0.1 \pm 1.6$ & $7.55 \pm 0.01$ & $6.42 \pm 0.01$ & $0.26 \pm 0.04$ & $0.21 \pm 0.04$ & 11.0 \\
\hline 2 & $5041 \pm 198$ & 5154 & 4.48 & $0.1 \pm 6.8$ & $7.52 \pm 0.04$ & $6.38 \pm 0.04$ & $0.52 \pm 0.11$ & $0.48 \pm 0.11$ & 12.4 \\
\hline 3 & $5077 \pm 188$ & 5162 & 4.48 & $1.6 \pm 1.1$ & $7.53 \pm 0.05$ & $6.40 \pm 0.05$ & $0.12 \pm 0.09$ & $0.10 \pm 0.09$ & 12.8 \\
\hline 2 & $5253 \pm 176$ & 5304 & $4.75 \pm 0.05$ & 1.1 & $7.56 \pm 0.02$ & $6.42 \pm 0.03$ & $0.54 \pm 0.09$ & $0.46 \pm 0.09$ & 10.5 \\
\hline 3 & $5190 \pm 120$ & 5260 & $4.68 \pm 0.05$ & 1.1 & $7.54 \pm 0.05$ & $6.40 \pm 0.04$ & $0.25 \pm 0.06$ & $0.24 \pm 0.06$ & 11.6 \\
\hline
\end{tabular}

Table 5. Best-fit depth-independent velocities.

\begin{tabular}{|c|c|c|c|c|}
\hline (1) & (2) & (3) & (4) & (5) \\
\hline $\begin{array}{c}\text { Star } \\
\text { \#Comp }\end{array}$ & $\begin{array}{c}v_{\text {mic,up }} \\
\left(\mathrm{km} \mathrm{s}^{-1}\right)\end{array}$ & $\begin{array}{c}v_{\text {mic,dn }} \\
\left(\mathrm{km} \mathrm{s}^{-1}\right)\end{array}$ & $\begin{array}{c}v_{\text {mac,rad }} \\
\left(\mathrm{km} \mathrm{s}^{-1}\right)\end{array}$ & $\begin{array}{c}v_{\text {mac,tan }} \\
\left(\mathrm{km} \mathrm{s}^{-1}\right)\end{array}$ \\
\hline$\alpha$ Cen A & \multirow{2}{*}{\multicolumn{2}{|c|}{$0.82 \pm 0.08$}} & & \\
\hline 1 & & & $4.0 \pm 0.7$ & $6.3 \pm 0.5$ \\
\hline 2 & $0.80 \pm 0.15$ & $0.90 \pm 0.20$ & $3.7 \pm 0.3$ & $4.0 \pm 0.3$ \\
\hline 3 & $0.84 \pm 0.04$ & $0.90 \pm 0.09$ & $3.4 \pm 0.3$ & $3.4 \pm 0.2$ \\
\hline$\alpha$ Cen B & \multirow{2}{*}{\multicolumn{2}{|c|}{$0.98 \pm 0.06$}} & & \\
\hline 1 & & & $1.0 \pm 3.1$ & $5.6 \pm 0.6$ \\
\hline 2 & $0.81 \pm 0.21$ & $1.07 \pm 0.18$ & $4.7 \pm 0.4$ & $0.4 \pm 1.1$ \\
\hline 3 & $0.62 \pm 0.08$ & $1.19 \pm 0.06$ & $4.6 \pm 0.4$ & $1.5 \pm 0.4$ \\
\hline $2^{a}$ & $0.52 \pm 0.44$ & $0.95 \pm 0.23$ & $3.6 \pm 0.8$ & $2.9 \pm 0.4$ \\
\hline $3^{a}$ & $0.38 \pm 0.54$ & $0.91 \pm 0.26$ & $3.9 \pm 3.4$ & $2.8 \pm 0.5$ \\
\hline $2^{b}$ & $0.77 \pm 0.23$ & $1.09 \pm 0.20$ & $4.6 \pm 0.6$ & $0.1 \pm 0.4$ \\
\hline $3^{b}$ & $0.79 \pm 0.27$ & $1.01 \pm 0.39$ & $3.3 \pm 0.7$ & $2.5 \pm 0.9$ \\
\hline
\end{tabular}

${ }^{a} \log g=4.48 ;{ }^{b} v \sin i=1.1 \mathrm{~km} \mathrm{~s}^{-1}$.

but less blue and red shifted absorptions in the up- and downflow areas. In other words, the combination of a blue- and redshifted absorption profile together with an unshifted profile, as in the 3-component model, allows larger shifts (or higher velocities) while reducing the need for a macro-turbulent broadening. The micro-turbulence velocity obtained from the inversions are higher in the downflows, in agreement with the solar findings by Nesis et al. (1992) that spectral lines formed there are broader.

The lower $\chi^{2}$ of the 1-component model is due mainly to the larger number of free parameters (since the $\lambda$ of each line is a free parameter) The best-fit net line shifts returned by the 1-component inversion reveal convective blueshifts of a few hundred $\mathrm{m} / \mathrm{s}$. Our values are smaller than the typical shifts of $0.2-1.2 \mathrm{~km} \mathrm{~s}^{-1}$ in the models of Dravins (1990), which is consistent with the way that the wavelength scale was determined.

A comparison with the 4-component model by Dravins (1990) is not straightforward. If we assume that his granular centers and granules correspond to our upflow component, his neutral areas to our horizontal flow region and his lanes to our downflow component then we end up with something like
30:55:15 for the ratios of areas harboring an upflow, horizontal flow and downflow, respectively, in reasonable agreement with our findings of 19:65:17. The depth independent velocities in the model of Dravins (1990) are difficult to compare with the velocity stratifications obtained from our models. Our velocities seem to correspond to smaller values, in particular when compared with his strong-line values.

\section{2. $\alpha$ Cen B}

$\alpha$ Cen B is significantly cooler and about one third smaller, but almost as massive as its companion. For this star we have carried out a larger number of inversions. First we discuss those for which we keep all parameters free. The effective temperatures obtained from the best-fit temperature stratifications and the ATLAS9 code are in good agreement with previous findings (see Tables 1 and 4). As in the case of $\alpha$ Cen A the $T_{\text {eff }}$ values returned by the 2- and 3-component models are significantly lower than that provided by the 1-component model. The temperature excess in the continuum forming layers of the component harboring an upflow is of similar magnitude as for $\alpha$ Cen A or the Sun (Fig. 4). According to the inversions, however, the temperature contrast between the up- and downflows shows practically no reversal, with upflows being hotter throughout most of the photosphere. As in the case of $\alpha$ Cen A the deduced abundances are lower by approximately 0.15 dex for the 2- and 3-component inversions than for the 1-component inversion.

There is a significant difference between the velocity structure found for this star and $\alpha$ Cen A or the Sun, in the sense that $\alpha$ Cen B exhibits a reduced overshoot. Above $z=100-150 \mathrm{~km}$ (with $z=0$ where $\tau_{5000}=1$ ) we found no significant vertical velocities (see Fig. 5 and compare with Fig. 4). This is mostly due to the smaller scale height on $\alpha$ Cen B. On the $\log \tau_{5000}$ scale the vertical velocities drop off like for $\alpha$ Cen A and vanish above $\log \tau_{5000}=-2$.

For $\alpha$ Cen $\mathrm{B}$ a much larger number of inversions have been carried out to test the influence of keeping individual parameters fixed. The motivation for these additional inversions came 
from the higher value of $\log g$ returned by the inversions than found previously, and by the fact that the inversions had difficulty determining $v \sin i$ accurately. The obtained surface acceleration seems to be independent of the number of components and always lies significantly above the values found in the literature. The results from inversions carried out with a fixed $\log g=4.48$, derived from Hipparcos data (Allende Prieto \& Lambert 1999), are shown in Fig. 6. The largest effect of this step is to lower $T_{\text {eff }}$ and, to a smaller extent, change the upand downflow stratifications. However, the large change seen when plotted vs. geometrical height is mainly due to the different scale-height produced by the smaller $\log g$. The other parameters, including the abundances, are not affected by this step.

The projected rotation velocity $v \sin i$, cannot be derived accurately by our inversions for this star. This is to be expected given the probably small value of this parameter (in the determination of Saar \& Osten (1997) $v \sin i$ is significant at less then the $1.5 \sigma$ level). The small line-broadening due to stellar rotation is not clearly distinguishable from the other line broadening mechanisms (micro- and macro-turbulence and instrumental broadening). Inversion carried out for a fixed $v \sin i=1.1 \mathrm{~km} \mathrm{~s}^{-1}$ are presented in Fig. 7 and Table 5. These results show that the temperature and velocity structure are not affected very much by such constraints. Neither are any of the other parameters, including the abundances. Replacing $v_{\text {inst }}=1.3 \mathrm{~km} \mathrm{~s}^{-1}$ by $1 \mathrm{~km} \mathrm{~s}^{-1}$ has a similarly small influence.

The granular up- and downflow areas are of similar size. For the downflow areas we found 10-24\% and for the upflow areas $12-26 \%$. The corresponding fractional areas in the 4-component model of Dravins (1990) for Arcturus (K1 III) would give $20 \%$ and $30 \%$, respectively. The convective blueshifts derived from the 1-component inversion and corrected by quantities obtained from the 2- and 3-component models are of order $0.2 \mathrm{~km} \mathrm{~s}^{-1}$ in good agreement with the findings of Dravins (1990) and synthetic profiles derived from numerical simulations (Nordlund \& Dravins 1990; Dravins \& Nordlund 1990).

The micro-turbulence and macro-turbulence parameters are associated with larger formal errors than for $\alpha$ Cen $\mathrm{A}$ (in particular the macro-turbulent velocities returned for the 2- and 3-component models show large differences). Taking into account the spread of results and larger estimated errors in values given in Table 5, however, we can say that the (depth-independent) micro-turbulence in the upflows is about $0.3 \mathrm{~km} \mathrm{~s}^{-1}$ lower than in the downflows and the tangential macro-turbulence is significantly lower than for $\alpha$ Cen A. It is also lower than the radial component of $v_{\mathrm{mac}}$. This fact indicates that the ratio of vertical to horizontal speed is larger on this star relative to the Sun or $\alpha$ Cen A (mind that the actual horizontal speeds are about 1.6 times larger then given by $v_{\text {mac,tan }}$ which must be interpreted as a spatial average over the line-ofsight component of velocities parallel to the surface but pointing in different directions around the axis of the granular cell, see Fig. 1). This ratio is proportional to the density scale height and inversely proportional to the size of the granular cells (see, e.g., Stein \& Nordlund 1998). Since the density scale height on $\alpha$ Cen $\mathrm{B}$ is smaller than on $\alpha$ Cen $\mathrm{A}$ a higher vertical to horizontal velocity ratio implies that the granules on $\alpha$ Cen B are smaller. This is in agreement with the results of simulations (e.g. Nordlund \& Dravins 1990).

\section{Conclusions}

We have carried out a spectroscopic analysis of the two brightest components of the $\alpha$ Cen system, named Toliman or Rigil Centaurus (arabic for "Foot of the Centaur"), based on multi-component inversions of 14 absorption line profiles. The temperature and velocity structure returned by the inversions clearly reveal hot areas harboring an upflow and areas with a cooler downflow for both $\alpha$ Cen A and its companion $\alpha$ Cen B.

In the case of $\alpha$ Cen $\mathrm{A}$, the multi-component inversions not only return the granulation properties that are close to previous findings (Dravins 1990) and very similar to the Sun (Frutiger et al. 2000), but are also able to give accurate values for fundamental parameters, like the total flux (effective temperature), the surface gravity or stellar rotation, that are in good agreement with values found with completely different methods. However, the elemental abundances are found to be up to 0.1 dex closer to solar values than previously found if we use our new multi-component models instead of an average 1-component model. This is in good agreement with recent results obtained by using 3-D hydrodynamic simulations (e.g. Asplund et al. 2005).

The results obtained for $\alpha$ Cen B are also promising. For example, besides returning the standard $T_{\text {eff }}$ value accurately, the multi-component inversions show that the vertical velocity stratification differs significantly from that in the Sun or $\alpha$ Cen A. There is also a hint (indirect) that the granules on $\alpha$ Cen B are smaller, which agrees well with numerical results. Hence, the inversion of cool-star spectra on the basis of multicomponent models is a promising way of efficiently and effectively determining both stellar fundamental parameters and granulation properties.

Nevertheless, some uncertainties remain. For example the sum of different broadening agents (macro-turbulence, stellar rotation, instrumental broadening) cannot be adequately extracted together with the temperature and flow stratification from the particular data used for this analysis. At the achieved spectral resolution, meaningful constraints on $v \sin i$ cannot be derived. The large $\log g$ values for $\alpha$ Cen B returned by the inversions also raise questions, although $\log g \approx 4.7$ appears to be a relatively robust result. The use of new spectra with higher spectral resolution $(>250000)$ may provide the necessary information and constraints in order to resolve these problems. As shown by Allende Prieto et al. (2001) the need for high signal-to-noise spectra is less critical.

The modeling of the granular flow field with 2 or 3 components has clear limitations. At disk center the granular upflows and intergranular downflows can be described reasonably well. Towards the limb, however, the horizontal velocities become important and may act as the dominant line broadening agent. We believe that the use of the radial-tangential function (Gray 1992) as presented in this work is a good first approximation to describe this behavior. But the simplifications made using such a description are obvious. Neither the depth-dependence 
of the vertical flow field is taken into account nor is the fact that a particular line of sight occasionally samples oppositely directed flows at the bottom and top of the atmosphere (see Fig. 1). To what extent such simplifications affect the results still remains to be determined.

Acknowledgements. We are grateful to Dr. Michael Lemke for his UNIX implementation of the ATLAS9 code by R. L. Kurucz, a part of the software library maintained by the CCP7 project. Special thanks go to Dr. Yvonne Unruh for her help in adapting the code for our DIGITAL UNIX machines. This research was supported by the Swiss Nationalfonds under NF grant No. 20-50464.97.

\section{References}

Allende Prieto, C., \& Lambert, D. L. 1999, A\&A, 352, 555

Allende Prieto, C., Ruiz Cobo, B., \& García López, R. J. 1998, ApJ, 502, 951

Allende Prieto, C., Barklem, P. S., Asplund, M., \& Ruiz Cobo, B., 2001, ApJ, 558, 830

Allende Prieto, C., Lambert, D. L., Tull, R. G., \& MacQueen, P. J. 2002, ApJ, 566, L93

Anstee, S. D., \& O'Mara, B. J. 1995, MNRAS, 276, 859

Asplund, M., Nordlund, A., Trampedach, R., \& Stein, R. F. 1999, A\&A, 346, L17

Asplund, M., Grevesse, N.., Sauval, A. J., Allende Prieto, C., \& Blomme, R. 2005, A\&A, 431, 693

Barklem, P. S., \& O’Mara, B. J. 1997, MNRAS, 290, 102

Barklem, P. S., O’Mara, B. J., \& Ross, J. E. 1998, MNRAS, 296, 1057

Bellot Rubio, L. R., Ruiz Cobo, B., \& Collados, M. 1998, ApJ, 506, 805

Bellot Rubio, L. R., Ruiz Cobo, B., \& Collados, M. 1999, A\&A, 341, L31

Cayrel, R., Faurobert-Scholl, M., Feautrier, N., Spielfieldel, A., \& Thévenin, F. 1996, A\&A, 312, 549

Chmielewski, Y., Friel, E., Cayrel de Strobel, G., \& Bentolila, C. 1992, A\&A, 263, 219

Dravins, D., Lindgren, L., \& Nordlund, Å. 1981, A\&A, 96, 345

Dravins, D. 1982, ARA\&A, 20, 61

Dravins, D. 1987a, A\&A, 172, 200

Dravins, D. 1987b, A\&A, 172, 211

Dravins, D. 1990, A\&A, 228, 218

Dravins, D., \& Nordlund, A. 1990, A\&A, 228, 203

Edvardsson, B. 1988, A\&A, 190, 148

Frutiger, C., Solanki, S. K., Fligge, M., \& Bruls, J. H. M. J. 2000 , A\&A, 358, 1109

Fuhr, J. R., Martin, G. A., \& Wiese, W. L. 1988, J. Phys. Chem. Ref. Data, 17, Suppl. 4

Furenlid, I., \& Meylan, T. 1990, ApJ, 350, 827

Gray, D. F. 1975, ApJ, 202, 148

Gray, D. F. 1992, The Observation and Analysis of Stellar Photospheres, 2nd ed. (Cambridge University Press)
Gray, D. F. 1998, in Tenth Cambridge Workshop on Cool Stars, Stellar Systems, and the Sun, ed. R. A. Donahu, \& J. A. Bookbinder, ASP Conf. Ser., 154, 10, 193

Gray, D. F. 1999, in Precise Stellar Radial Velocities, ed. J. B. Hearnshaw, \& C. D. Scarfe, ASP Conf. Ser., 185, 243

Gray, D. F., \& Toner, C. G. 1985, Publ. Astron. Soc. Pac., 97, 543

Grevesse, N., \& Sauval, A. J. 1999, A\&A, 347, 348

Hallam, K. L., Altner, B., \& Endal, A. S. 1991, ApJ, 372, 610

Hamilton, D., \& Lester, J. B. 1998, in Tenth Cambridge Workshop on Cool Stars, Stellar Systems, and the Sun, ed. R. A. Donahu \& J. A. Bookbinder, ASP Conf. Ser., 154, 1594

Hanslmeier, A., Mattig, W., \& Nesis, A. 1991, A\&A, 248, 232

Jay, J. E., Guinan, E. F., Morgan, N. D., Messina, S., \& Jassour, D. 1996, in Bull. Am. Astron. Soc., 189, 12004

Kurucz, R. L. 1970, Atlas: A Computer Program for Calculating Model Stellar Atmospheres, SAO Special Report, Cambridge: Smithsonian Astrophysical Observatory

Kurucz, R. L., \& Bell, B. 1995, Harvard-Smithsonian Center for Astrophysics, CD-ROM No. 23

Landi Degl'Innocenti, E. 1976, A\&A, 25, 379

Livingston, W., Wallace, L., Huang, Y., \& Moise, E. 1999, in High Resolution Solar Physics: Theory, Observations, and Techniques, ed. T. R. Rimele, K. S. Balasubramaniam, \& R. R. Radick, ASP Conf. Ser., 183, 494

Miller, M. H., Wilkerson, T. D., Roig, R. A., \& Bengston, R. D. 1974, J. Phys. Chem. Ref. Data, A 9, 2312

Nave, G., Johansson, S., Learner, R. C. M., Thorne, A. P., \& Brault, J. W. 1994, ApJS, 94, 221

Neckel, D. 1994, in The Sun as a Variable Star, ed. J. Pap, C. Fröhlich, H. Hudson, S. Solanki (Cambridge: Cambridge, Univ. Press), IAU Coll., 143, 37

Neckel, D. 1999, unpublished spectral atlas

Nesis, A., Hanslmeier, A., Hammer, R., et al. 1992, A\&A, 253, 561

Nesis, A., Hanslmeier, A., Hammer, R., et al. 1993, A\&A, 279, 599

Nordlund, Å., \& Dravins, D. 1990, A\&A, 228, 155

Nordlund, Å., \& Stein, R. F. 1996, in Proc. of the 32nd Liège Int. Astrophys. Coll., ed. Noels et al., Université de Liège, Institut d'Astrophysique, 75

Ploner, S. R. O., Solanki, S. K., \& Gadun, A. S. 2000, A\&A, 356, 1050

Rüedi, I., Solanki, S. K., Mathys, G., \& Saar, S. H. 1997, A\&A, 318, 429

Ruiz Cobo, B., \& Del Toro Iniesta, J. C. 1992, ApJ, 398, 375

Saar, S. H., \& Osten, R. A. 1997, MNRAS, 284, 803

Schnabel, R., Kock, M., \& Holweger, H. 1999, A\&A, 342, 610

Soderblom, D. R. 1986, A\&A, 158, 273

Spruit, H. C., \& Nordlund, Å., \& Title, A. M. 1990, ARA\&A, 28, 263

Stein, R. F., \& Nordlund, Å. 1998, ApJ, 499, 914

Sugar, J., \& Corliss, C. 1985, J. Phys. Chem. Ref. Data, 14, Suppl. 2

Unsöld, A. 1955, Physik der Sternatmosphären, 2nd ed. (Berlin: Springer Verlag) 